\title{
Neutrophil-to-Lymphocyte, Lymphocyte-to- Monocyte, and Platelet-to-Lymphocyte Ratios: Prognostic Significance in COVID-19
}

\author{
Shivakumar BG ${ }^{1}$, Siddharth Gosavi ${ }^{1}$, Amogh Ananda Rao ${ }^{1}$, Shashank Shastry ${ }^{1}$, Sharanya C. Raj ${ }^{1}$, \\ Anirudha Sharma ${ }^{1}$, Ashutosh Suresh ${ }^{1}$, Rakesh Noubade ${ }^{1}$ \\ 1. Internal Medicine, Jagadguru Jayadeva Murugarajendra Medical College, Davangere, IND
}

Corresponding author: Siddharth Gosavi, ramshyamsid@gmail.com

\section{Abstract \\ Background and objective}

Neutrophils are primarily responsible for activating the immune system, and systemic inflammation destroys CD4+ T lymphocytes and increases suppressor CD8+ T lymphocytes, thereby leading to an increased neutrophil-to-lymphocyte ratio (NLR). An increase in the apoptosis of lymphocytes leads to lymphopenia and elevated thrombopoietin (THPO) promotes megakaryocyte production. The reflections of these inflammatory changes can be vital in gauging the progression of the disease.

This study aimed at examining the prognostic value of normal and derived neutrophil-to-lymphocyte, lymphocyte-to-monocyte, platelet-to-lymphocyte, and mean platelet volume (MPV)-to-platelet count ratios in patients with coronavirus disease 2019 (COVID-19).

\section{Methodology}

This was a retrospective cross-sectional study conducted in the wards of Chigateri General Hospital, Davangere for a period of two months. Complete blood count was ordered for all patients at the time of admission along with confirmation of the disease by reverse transcription-polymerase chain reaction (RTPCR).

\section{Results}

The final study population consisted of 100 patients. The mean age of patients who survived (43 years) was significantly lower than the mean age of non-survivors (59.1 years), with a p-value of $<0.001$. NLR was raised in $60 \%$ of the population and was significantly higher in patients who survived the disease, with a p-value of 0.004. The platelet-to-lymphocyte ratio (PLR) also followed a similar trend with a p-value of 0.017 . Even though the lymphocyte-to-monocyte ratio (LMR) also mimicked the trend, the statistical association was not significant (p-value: 0.09). The derived NLR and MPV-to-platelets ratios were not found to be significantly associated with mortality in this study.

Review began 12/15/2020 Review ended 01/05/2021 Published 01/11/2021

\section{() Copyright 2021}

$B G$ et al. This is an open access article distributed under the terms of the Creative Commons Attribution License CC-BY 4.0., which permits unrestricted use, distribution, and reproduction in any medium, provided the original author and source are credited.

\section{Discussion}

Younger patients had better clinical outcomes in our study population compared to the geriatric age group. A significant correlation between LMR and mortality was observed when a cut-off of 2.5 was considered as a differentiating benchmark.

Conflicting trends were observed in NLR and PLR in our study; however, LMR was in accordance with most other studies. The phase that a patient is in with regard to the natural history of the disease also influences the blood cell ratios. Nonetheless, all three ratios can be used as crucial screening and prognostic tools as they are readily available with the help of a complete hemogram. This is an investigation modality that is widely accessible even in remote areas and resource-limited settings.

\section{Conclusion}

These hematological ratios can facilitate in categorizing the disease severity and progression in patients, thereby enabling us to make appropriate and informed clinical decisions. Since the second wave of the novel coronavirus is on the verge of arrival, it is imperative to channel resources for the patients early in their disease course to ultimately prevent complications and reduce mortality.

Categories: Internal Medicine, Infectious Disease, Hematology Keywords: covid-19, prognostic markers, complete blood count 
Neutrophils constitute the majority of the leukocytes and are primarily responsible for activating the immune system by migrating from the venous system. Free oxygen radicals that can damage the nuclear material of the cell are thereby released. Viral antigens are exposed and cell-specific and humoral immunities are stimulated by an antibody-dependent cell-mediated cell. There is a growing interaction with molecules like vascular endothelial growth factor (VEGF), interleukin-6, interleukin-8, tumor necrosis factor-alpha (TNF- $\alpha$ ), interferon-gamma, and granulocyte colony-stimulating factor. VEGF-A and VEGF-C are particularly notorious in the novel coronavirus, severe acute respiratory syndrome coronavirus-2 (SARSCoV-2) [1]. The immune response is massively dependent on lymphocytes. On the other hand, systemic inflammation destroys CD4+ T lymphocytes and increases suppressor CD8+ T lymphocytes, thereby leading to an increased neutrophil-to-lymphocyte ratio (NLR). Formerly, NLR was mainly used in oncological conditions, autoimmune diseases, and bacteriological infections [2,3]. However, in a study conducted by Yang et al., it was found to be an independent prognostic factor in patients with coronavirus disease 2019 (COVID-19). This reinforces the belief in the relationship between hyper-inflammation and SARS-CoV-2 [4]. In another study conducted by Ciccullo et al, an NLR of greater than four was seen as a predictor of the admission of COVID-19 patients to the intensive care unit. The duration of hospital stay was prolonged, and the time lag for nucleic acid results to become negative was increased. It is a valuable tool for screening critically ill patients with confirmed SARS-CoV-2 infection, as it can contribute to the evaluative acumen of the physician [5].

T helper cells induce the production of cytokines such as interleukin-17 through the nuclear factor kappalight-chain-enhancer of activated B cells (NF-kB) signaling pathway, leading to increased aggregation of monocytes. SARS-CoV-2 infects circulating immune cells and increases apoptosis of lymphocytes, leading to lymphopenia. A lower ratio of circulating lymphocytes to monocytes (LMR) predicts severe and extremely severe COVID-19 as the clearance of the virus is delayed due to lymphopenia and also a decrease in CD4+ T cells. Both a rise or fall in lymphocyte levels is an extremely crucial prognostic indicator of mortality in COVID-19[6].

Platelets play a crucial role in homeostasis, coagulation, vascular integrity maintenance, angiogenesis, innate immunity, and inflammatory response. In a state of inflammation, interleukin-6 promotes megakaryocyte production by stimulating an increase of thrombopoietin (THPO) level. Platelet count is a sensitive reflection of the body's infection and inflammatory state. In a study conducted by Qu et al., it was found that platelets increased first and then decreased in several of their patients during treatment. Coronavirus invades bone stromal cells, leading to hematopoietic inhibition [7]. Also, mature megakaryocytes release platelets in the lung. Therefore, thrombocytopenia and lung damage go hand in hand. Extensive alveolar damage is also observed. Lung damage results in pulmonary endothelial injury, which in turn leads to activation, aggregation, and retention of platelets in the lung. It is followed by the formation of a thrombus, which leads to the depletion of platelets. The cytokine storm is responsible for worsening the inflammation in the patient as it causes increased secretion of Th2 cytokines that inhibit Th1 cytokines, such as interleukin- 4 and interleukin-10. Tracheal intubation and deep vein catheterization are potential factors that affect platelet changes. Platelet-released platelet factor- 4 can prevent agglutinin-A from inhibiting lymphocyte generation; activated platelets enhance lymphocyte adhesion to the endothelium, thereby promoting lymphocyte homing in endothelial veins and migration to inflammatory sites. It indicates both aggregation and inflammatory pathways, thereby showing the level of inflammation even during the course of treatment. This also correlated with an increase in the stay at the hospital. Therefore, the platelet-to-lymphocyte ratio (PLR) provides a reliable reflection of the extent of cytokine inflammation and can be employed for monitoring purposes in patients with COVID-19 [8].

The mean platelet volume (MPV)-to-platelet count ratio has been recently proposed as a prognostic marker in SARS-CoV-2. In inflammation, platelet production increases due to the increased synthesis of THPO, which is mediated through cytokines. MPV reflects the proliferation of megakaryocytes and platelet production in the bone marrow. There is an increased expression of young platelets in the bloodstream, leading to increased MPV. Decreasing platelet count forces the body's immune system to stimulate megakaryocytes to produce a large number of platelets, thereby increasing MPV. Adverse prognosis is due to the increased oxidative stress, thrombosis, and apoptosis in activated platelets [9]. It symbolizes a higher risk of poor outcomes in COVID-19 patients.

These four ratios have been scrutinized in great detail in SARS-CoV-2 patients in other countries that have a higher financial and resource allocation for healthcare. However, in resource-limited settings or where a health facility is functioning beyond its capacity, investigations like interleukin-6, interleukin-10, and other special platelet tests cannot be routinely performed. Therefore, by employing a simple complete blood count, we aimed to study the importance of derived NLR along with the other four ratios in patients with SARS-CoV-2. We also aimed to investigate their relationship with mortality in patients with COVID-19.

\section{Materials And Methods}

This was a retrospective cross-sectional study conducted at the Chigateri General Hospital, Davangere, for a period of two months: July and August of 2020. We commenced the study after obtaining approval from the Institutional Ethics Committee. 


\section{Cureus}

Cases were defined based on the interim guidelines issued by the Ministry of Health and Family Welfare, Government of India. Admitted patients with the respiratory rate exceeding 24 cycles per minute and oxygen saturation below 94\% without oxygen supplementation (falling under Category B and Category C) were included in the study. A complete hemogram was ordered within one hour of admission, before the initiation of treatment. The diagnosis of COVID-19 was confirmed with reverse transcription-polymerase chain reaction (RT-PCR) for viral RNA. Patients receiving mechanical ventilation, patients on tocilizumab, pirfenidone, azathioprine, and cyclophosphamide, and those with a previous diagnosis of malignancy were excluded from the study. Patients with other febrile illnesses like dengue, malaria, leptospirosis, and rickettsial fevers were also excluded from the study. Figure 1 presents the treatment protocol followed in the care of all patients in the study population.

Treatment Protocol for COVID-19 Patients falling under Categories B and C

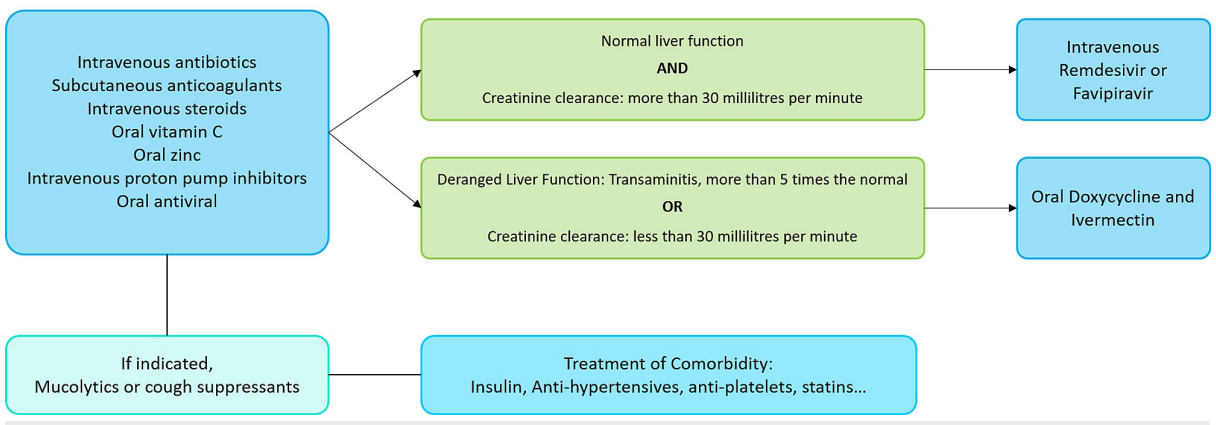

FIGURE 1: Treatment protocol for COVID-19 patients falling under Categories $B$ and $C$

Categories B and C were defined by the interim guidelines issued by the Ministry of Health and Family Welfare, Government of India

COVID-19: coronavirus disease 2019

Various ratios that are known to have prognostic value and examined in this study are presented in Table 1 . The laboratory records were collected and screened to match the criteria of our study. Data analysis was performed using IBM SPSS Statistics version 27 (IBM, Armonk, NY).

Parameter
Neutrophil-to-lymphocyte ratio
Derived neutrophil-to-lymphocyte ratio
Lymphocyte-to-monocyte ratio
Platelet-to-lymphocyte ratio
Mean platelet volume-to-platelet count
ratio

\section{Acronym Formula}

N/L (Absolute neutrophil count) / (absolute lymphocyte count)

Derived (Absolute neutrophil count) / (total leukocyte count - absolute neutrophil N/L count)

L/M (Absolute lymphocyte count) / (absolute monocyte count)

PIt/L (Platelet count) / (absolute lymphocyte count)

MPV/PIt (Mean platelet volume) / (platelet count)

\section{TABLE 1: Definition of prognostic markers}

Results were presented as mean, standard deviation, and range values for continuous measurements and frequencies as numbers and percentages. The unpaired t-test was used to compare the means between any two groups. Whenever measurements displayed non-Gaussian distribution, the Mann-Whitney U test was used as an alternative to the t-test. Categorical data were analyzed by the chi-squared test. The odds ratio was used to compute the odds of occurrence of an event (mortality) using bio-marker. Diagnostic validity tests were also performed to assess the utility of each parameter. Receiver operator characteristic (ROC) curves were used to compare the different parameters. A p-value of 0.05 or less was considered statistically significant.

\section{Results}




\section{Cureus}

The final study population consisted of 100 patients. Table 2 presents the demographic description of the sample. The mean age was $47.1 \pm 14.8$ years (range: 20 - 78 years). There were 57 males and 43 females were; 75 patients survived the viral disease, and 25 patients succumbed to the virus.

The mean age of patients who survived (43 years) was significantly lower than that of non-survivors (59.1 years), with a p-value of $<0.001$.

\begin{tabular}{|c|c|c|c|c|c|}
\hline \multicolumn{6}{|c|}{ Demographic characteristics } \\
\hline \multicolumn{2}{|c|}{ Number of cases } & All cases & Non-survivors & Survivors & Non-survivors vs. survivors \\
\hline \multirow{2}{*}{ Age (years) } & Mean \pm SD & $47.1 \pm 14.8$ & $59.1 \pm 11.5$ & $43.0 \pm 13.6$ & \multirow{2}{*}{$t=5.31 ; p<0.001(H S)$} \\
\hline & Range & $20-78$ & $40-78$ & $20-71$ & \\
\hline \multirow{2}{*}{ Sex } & Male & 57 & 13 & 44 & \multirow{2}{*}{$x^{2}=0.34 ; p=0.56(N S)$} \\
\hline & Female & 43 & 12 & 31 & \\
\hline
\end{tabular}

\section{TABLE 2: Descriptive information of study subjects}

The mean age of patients who did not survive the disease was significantly higher $(p<0.001)$

SD: standard deviation; HS: highly significant; NS: not significant

Figure 2 depicts the presenting complaints of all the patients. Breathlessness was the most frequent complaint, followed by productive and dry cough, and fever. The duration of symptoms ranged from one to five days. Table 3 presents a comparison of the symptoms between survivors and non-survivors.

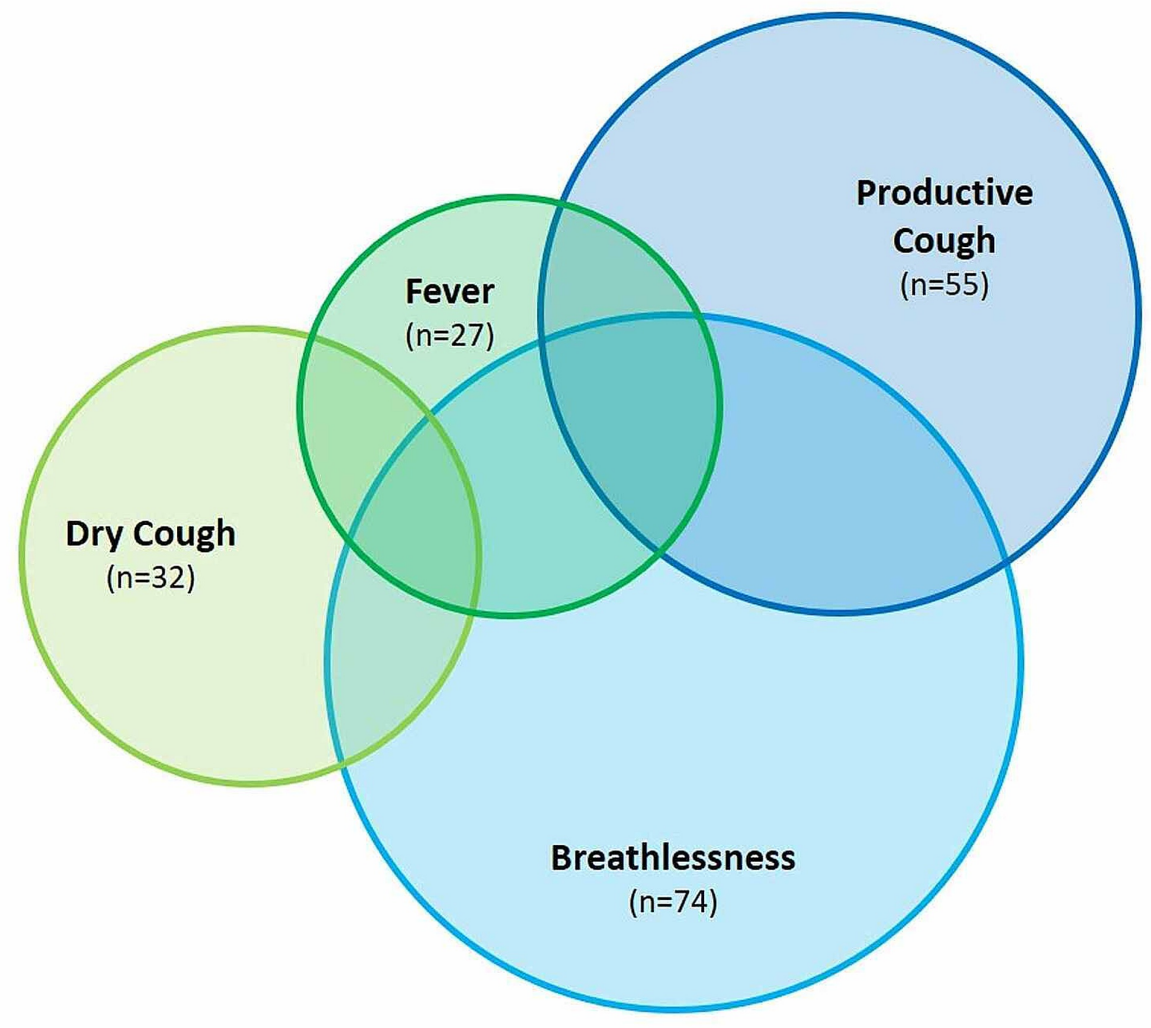

FIGURE 2: Representation of symptomatology 


\section{Cureus}

\begin{tabular}{|c|c|c|}
\hline Symptom & Non-survivors $(n=25)$ & Survivors $(n=75)$ \\
\hline Fever & 10 & 15 \\
\hline Dry cough & 9 & 23 \\
\hline Productive cough & 12 & 43 \\
\hline Breathlessness & 17 & 57 \\
\hline
\end{tabular}

\section{TABLE 3: Comparison of symptoms between survivors and non-survivors}

Figure 3 documents the presence of comorbidities in the study population. Diabetes mellitus was the most common accompanying morbidity, followed by hypertension and other conditions. Table 4 provides a comparison with respect to comorbidities between patients who survived the disease and those who succumbed to the disease.

\section{Distribution of Comorbidities}

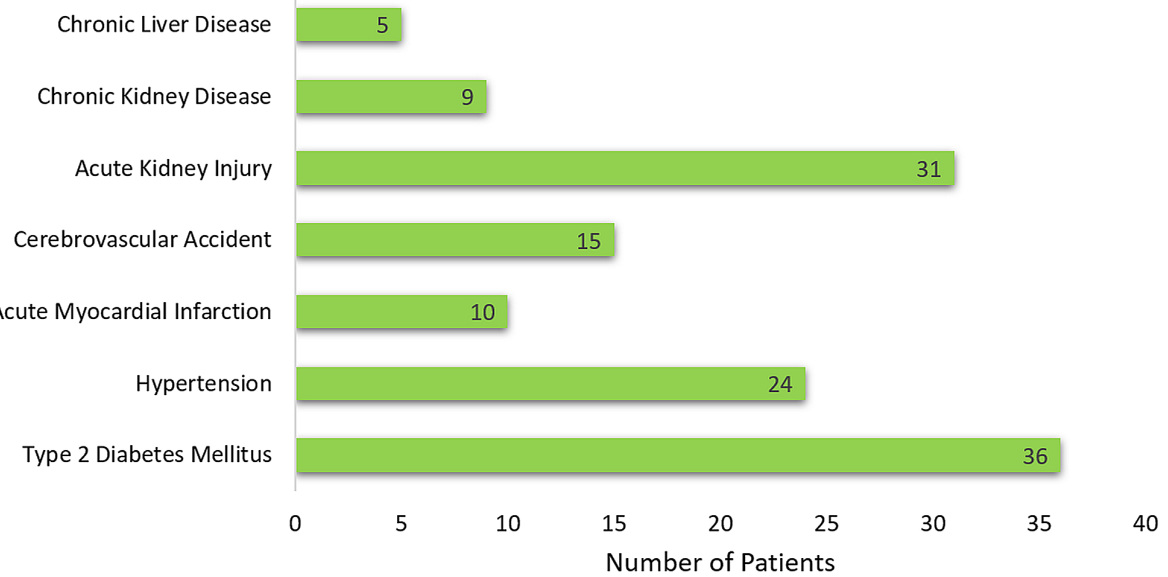

FIGURE 3: Distribution of comorbidities in the study population

\section{Comorbidity}

Type 2 diabetes mellitus

Hypertension

Acute myocardial infarction

Cerebrovascular accident

Acute kidney injury

Chronic kidney disease

Chronic liver disease

\section{Non-survivors}

19

5

5

3

4
Survivors

17

19

5

12

27

5

4

TABLE 4: Comparison of comorbid conditions between survivors and non-survivors

Table 5 summarizes the distribution of various cell lines included in the calculation of prognostic parameters investigated in this study. 


\section{Cureus}

\begin{tabular}{|c|c|c|c|c|c|}
\hline Cell line & Mean & Standard deviation & Median & Minimum & Maximum \\
\hline Total leukocyte count (cells/cumm) & 9,775 & $5,698.1$ & 8,300 & 800 & 31,400 \\
\hline Platelet count (cells/cumm) & 22,849 & $9,646.2$ & 22,200 & 2,300 & 46,700 \\
\hline Absolute neutrophil count (cells/cumm) & $7,063.1$ & $5,089.8$ & $5,457.6$ & 247.5 & 29,257 \\
\hline Absolute lymphocyte count (cells/cumm) & $1,471.3$ & 905.7 & $1,438.8$ & 102.4 & $4,647.2$ \\
\hline Absolute monocyte count (Cells/cumm) & 884.5 & 745.1 & 671.5 & 22.5 & 3,799 \\
\hline
\end{tabular}

TABLE 5: Descriptive statistics of cell lines used to compute prognostic parameters

Table 6 presents the descriptive statistics of the prognostic parameters for correlation with disease outcomes. The NLR was raised in $60 \%$ of the population.

\begin{tabular}{|c|c|c|c|c|}
\hline Parameter & Mean \pm SD & Median & Minimum & Maximum \\
\hline Derived N/L & $3.49 \pm 3.02$ & 2.69 & 0.45 & 20.28 \\
\hline N/L & $7.9 \pm 8.9$ & 4.1 & 0.99 & 39.71 \\
\hline L/M & $3.06 \pm 4.15$ & 2.07 & 0.16 & 27.71 \\
\hline Plt/L & $27.0 \pm 42.6$ & 18.5 & 1.4 & 300.8 \\
\hline MPV/PIt & $0.00052 \pm 0.0005$ & 0.0004 & 0.0002 & 0.0037 \\
\hline
\end{tabular}

\section{TABLE 6: Descriptive statistics on test measurements}

SD: standard deviation; N/L: neutrophil-to-lymphocyte ratio; L/M: lymphocyte-to-monocyte ratio; PIt/L: platelet-to-lymphocyte ratio; MPV/PIt: mean platelet volume-to-platelet count ratio

Table 7 compares the prognostic markers between survivors and non-survivors. The NLR was significantly higher in patients who survived the disease, with a p-value of 0.004. PLR also followed a similar trend with a $\mathrm{p}$-value of 0.017. Even though LMR also mimicked the trend, the statistical association was not significant (p-value: 0.09).

\begin{tabular}{|l|lllll|l|}
\hline \multirow{2}{*}{ Parameter } & \multicolumn{2}{l}{ Non-survivors $(\mathbf{n}=\mathbf{2 5})$} & \multicolumn{2}{l|}{ Survivors $(\mathbf{n}=\mathbf{7 5})$} & \multicolumn{2}{l|}{ Non-survivors vs. survivors } \\
\cline { 2 - 3 } & Mean & SD & Mean & SD & $\mathrm{t}$ & P-value \\
\hline Derived N/L & 2.65 & 1.93 & 3.77 & 3.27 & -1.61 & 0.11 (NS) \\
N/L & 4.87 & 3.7 & 8.88 & 3.84 & 2.96 & 0.004 (S) \\
L/M & 2.17 & 2.13 & 3.35 & 4.61 & - & 0.09 (NS) \\
PIt/L & 15.43 & 9.44 & 30.52 & 48.21 & - & 0.017 (S) \\
MPV/PIt & 0.00028 & 0.00046 & 0.00047 & 0.00072 & - & 0.14 (NS) \\
\hline
\end{tabular}

\section{TABLE 7: Comparison of test parameters between non-survivors and survivors}

$\mathrm{N} / \mathrm{L}$ and $\mathrm{PIt} / \mathrm{L}$ differ significantly between survivors and non-survivors, with $\mathrm{p}$-values of 0.004 and 0.017 respectively

SD: standard deviation; N/L: neutrophil-to-lymphocyte ratio; L/M: lymphocyte-to-monocyte ratio; PIt/L: platelet-to-lymphocyte ratio; MPV/PIt: mean platelet volume-to-platelet count ratio; S: significant; NS: not significant 


\section{Cureus}

cut-off value for each parameter was considered as a suitable value around the median, which showed high sensitivity and accuracy in the prediction of mortality. The statistical correlation between the clinical outcomes and the cut-off values has been shown.

\begin{tabular}{|c|c|c|c|c|c|c|c|c|}
\hline \multicolumn{9}{|c|}{ Ratio-wise distribution of cases and their significance } \\
\hline \multirow{2}{*}{ Test parameter } & \multirow{2}{*}{ Cut-off value } & \multicolumn{2}{|c|}{ Non-survivors $(n=25)$} & \multicolumn{2}{|c|}{ Survivors $(n=75)$} & \multicolumn{2}{|c|}{ Non-survivors vs. survivors } & \multirow{2}{*}{ Odds ratio $(95 \% \mathrm{Cl})$} \\
\hline & & Number & $\%$ & Number & $\%$ & $x^{2}$ & P-value & \\
\hline \multirow{2}{*}{$N / L$} & $\leq 4.7$ & 18 & 72.0 & 37 & 49.3 & \multirow{2}{*}{3.89} & \multirow{2}{*}{$0.049(\mathrm{~S})$} & \multirow{2}{*}{$2.6(1.0-7.1)$} \\
\hline & $>4.7$ & 7 & 28.0 & 38 & 50.7 & & & \\
\hline \multirow[b]{2}{*}{ L/M } & $\leq 2.5$ & 22 & 88.0 & 41 & 54.7 & \multirow{2}{*}{8.94} & \multirow{2}{*}{$0.003(\mathrm{~S})$} & \multirow{2}{*}{$6.1(1.7-22.1)$} \\
\hline & $>2.5$ & 3 & 12.0 & 34 & 45.3 & & & \\
\hline \multirow{2}{*}{$\mathrm{Plt} / \mathrm{L}$} & $\leq 20.0$ & 20 & 80.0 & 43 & 57.3 & \multirow{2}{*}{4.13} & \multirow{2}{*}{$0.04(\mathrm{~S})$} & \multirow{2}{*}{$3.0(1.0-8.8)$} \\
\hline & $>20.0$ & 5 & 20.0 & 32 & 42.7 & & & \\
\hline
\end{tabular}

\section{TABLE 8: Ratio-wise distribution of cases and their significance in differentiating the final} outcome

N/L: neutrophil-to-lymphocyte ratio; L/M: lymphocyte-to-monocyte ratio; PIt/L: platelet-to-lymphocyte ratio; S: significant

Diagnostic test validity of NLR, LMR, and PLR was analyzed regarding the prediction of mortality of COVID19 using a ROC curve (Table 9, Figure 4). These tests have a high sensitivity and high negative predictive value. These parameters can be used to rule out the possibility of death when these values are high.

\begin{tabular}{|c|c|c|c|}
\hline \multirow{2}{*}{ Parameters } & N/L & L/M & PIt/L \\
\hline & $\leq 4.7$ & $\leq 2.5$ & $\leq 20.0$ \\
\hline Sensitivity & $72 \%$ & $88 \%$ & $80 \%$ \\
\hline Specificity & $51 \%$ & $45 \%$ & $43 \%$ \\
\hline PPV & $33 \%$ & $35 \%$ & $32 \%$ \\
\hline NPV & $84 \%$ & $92 \%$ & $87 \%$ \\
\hline Accuracy & $56 \%$ & $56 \%$ & $52 \%$ \\
\hline
\end{tabular}

\section{TABLE 9: Diagnostic validity tests for predicting mortality using various significant parameters}

N/L: neutrophil-to-lymphocyte ratio; L/M: lymphocyte-to-monocyte ratio; PIt/L: platelet-to-lymphocyte ratio; PPV: positive predictive value; NPV: negative predictive value 
ROC Curves

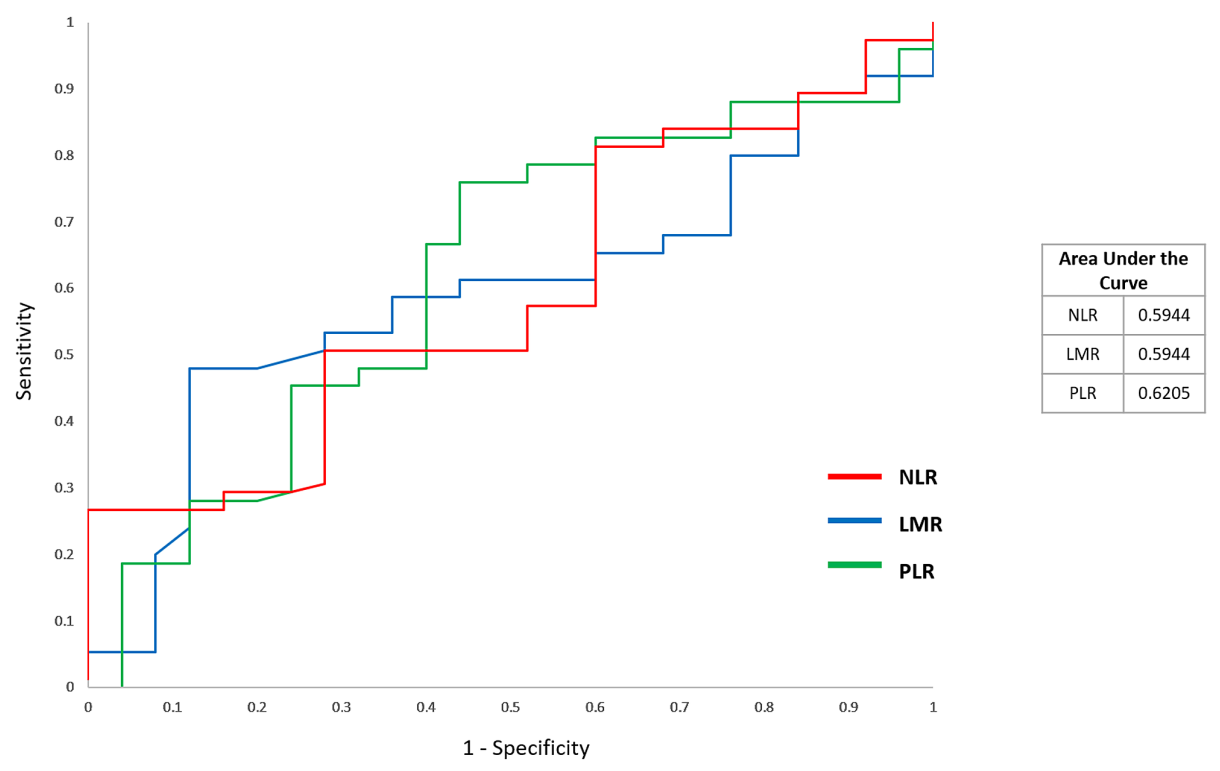

FIGURE 4: ROC curve and area under the curve

ROC: receiver operator characteristic; NL: neutrophil-to-lymphocyte; LM: lymphocyte-tomonocyte; PItL: platelet-to-lymphocyte

\section{Discussion}

Younger patients had better clinical outcomes in our study population compared to the geriatric age group. The difference in mean age was 16 years between survivors and non-survivors. This observation is consistent with other studies that have implicated old age to be a factor contributing to worse clinical course and mortality $[10,11]$.

NLR was higher than normal in the majority of the patients. However, it was 1.8 times higher among survivors in our study. This is in stark contrast with the trend observed in a study conducted by Usul et al., wherein NLR was was found to be significantly high in critically ill COVID-19 patients compared to the control population [12]. LMR was also higher in survivors but the association was not statistically significant. The LMR decreased in non-survivors in our study, which is in agreement with studies conducted by Lissoni et al. and Zhang et al. [13,14]. However, a significant correlation was observed with mortality when a cut-off of 2.5 was considered as a differentiating benchmark. PLR was twice as high among patients who survived compared to the non-survivors in our study. This observation also was in contrast with a study conducted by Zhao et al., wherein the PLR increased significantly in critically ill patients [15]. The derived NLR and MPV-to-platelets ratio were not found to be significantly associated with mortality in this study.

Conflicting trends were observed in NLR and PLR in our study; however, LMR was in accordance with most other published studies. The point of observation in our study was that at the time of admission and followup, investigations were not performed in all patients due to financial and logistic constraints. The phase that a patient is in with regard to the natural history of the disease also influences the blood cell ratios. Since the objective was to derive prognostic insights from the ratios, all the investigations were ordered at the time of admission to the hospital.

All three ratios can be used as crucial screening and prognostic tools as they are readily available with the help of a complete hemogram. This is an investigation that is widely accessible even in remote areas and resource-limited settings. This is a cost-effective tool in situations where logistics and finances are constrained. In our study, all three ratios were found to have high sensitivity and high negative predictive values, helping to gauge the progression of disease early in its course.

\section{Limitations}

This study has some limitations. Serial investigations could not be performed due to financial and logistical constraints related to the patients. The sample size was small and, hence, further, larger studies are warranted. Patients could not be followed up after discharge for persisting symptoms or post-COVID sequelae as the majority were anxious and apprehensive about returning to the hospital. 


\section{Conclusions}

These hematological ratios that we discussed in our study can facilitate in categorizing the disease severity and progression in patients, thereby enabling us to make appropriate and informed clinical decisions. Since the second wave of the novel coronavirus is on the verge of arrival, it is imperative to channel resources for the patients early in their disease course to ultimately prevent complications and reduce mortality.

\section{Additional Information \\ Disclosures}

Human subjects: Consent was obtained by all participants in this study. Institutional Ethics Committee, Chigateri General Hospital issued approval N/A. This study was approved by the Institutional Ethics Committee of Chigateri General Hospital. Animal subjects: All authors have confirmed that this study did not involve animal subjects or tissue. Conflicts of interest: In compliance with the ICMJE uniform disclosure form, all authors declare the following: Payment/services info: All authors have declared that no financial support was received from any organization for the submitted work. Financial relationships: All authors have declared that they have no financial relationships at present or within the previous three years with any organizations that might have an interest in the submitted work. Other relationships: All authors have declared that there are no other relationships or activities that could appear to have influenced the submitted work.

\section{References}

1. Kim SL, Lee ST, Trang KT, et al.: Parthenolide exerts inhibitory effects on angiogenesis through the downregulation of VEGF/VEGFRs in colorectal cancer. Int J Mol Med. 2014, 33:1261-7. 10.3892/ijmm.2014.1669

2. Ying HQ, Deng QW, He BS, et al.: The prognostic value of preoperative NLR, d-NLR, PLR and LMR for predicting clinical outcome in surgical colorectal cancer patients. Med Oncol. 2014, 31:305. 10.1007/s12032014-0305-0

3. Uslu AU, Küçük A, Şahin A, et al.: Two new inflammatory markers associated with Disease Activity Score-28 in patients with rheumatoid arthritis: neutrophil-lymphocyte ratio and platelet-lymphocyte ratio. Int J Rheum Dis. 2015, 18:731-5. 10.1111/1756-185X.12582

4. Yang AP, Liu JP, Tao WQ, Li HM: The diagnostic and predictive role of NLR, d-NLR and PLR in COVID-19 patients. Int Immunopharmacol. 2020, 84:106504. 10.1016/j.intimp.2020.106504

5. Ciccullo A, Borghetti A, Zileri Dal Verme L, et al.: Neutrophil-to-lymphocyte ratio and clinical outcome in COVID-19: a report from the Italian front line. Int J Antimicrob Agents. 2020, 56:106017. 10.1016/j.ijantimicag.2020.106017

6. Liu G, Zhang S, Hu H, Liu T, Huang J: The role of neutrophil-lymphocyte ratio and lymphocyte-monocyte ratio in the prognosis of type 2 diabetics with COVID-19. Scott Med J. 2020, 65:154-60. 10.1177/0036933020953516

7. Zhang Y, Zeng X, Jiao Y, Li Z, Liu Q, Ye J, Yang M: Mechanisms involved in the development of thrombocytopenia in patients with COVID-19. Thromb Res. 2020, 193:110-5. 10.1016/j.thromres.2020.06.008

8. Qu R, Ling Y, Zhang YH, et al.: Platelet-to-lymphocyte ratio is associated with prognosis in patients with coronavirus disease-19. J Med Virol. 2020, 92:1533-41. 10.1002/jmv.25767

9. Zhong Q, Peng J: Mean platelet volume/platelet count ratio predicts severe pneumonia of COVID-19 (Epub ahead of print). J Clin Lab Anal. 2020, e23607. 10.1002/jcla.23607

10. Yanez ND, Weiss NS, Romand JA, Treggiari MM: COVID-19 mortality risk for older men and women . BMC Public Health. 2020, 20:1742. 10.1186/s12889-020-09826-8

11. Bonanad C, García-Blas S, Tarazona-Santabalbina F, et al.: The effect of age on mortality in patients with COVID-19: a metanalysis with 611,583 subjects. J Am Med Dir Assoc. 2020, 21:915-8. 10.1016/j.jamda.2020.05.045

12. Usul E, Şan İ, Bekgöz B, Şahin A: Role of hematological parameters in COVID-19 patients in the emergency room. Biomark Med. 2020, 14:1207-15. 10.2217/bmm-2020-0317

13. Lissoni P, Rovelli F, Monzon A: Evidence of abnormally low lymphocyte-to-monocyte ratio in COVID-19induced severe acute respiratory syndrome. J Immunol Allergy. 2020, 1:549. 10.37191/Mapsci-2582-6549$1(2)-011$

14. Zhang H, Cao X, Kong M, et al.: Clinical and hematological characteristics of 88 patients with COVID-19. Int J Lab Hematol. 2020, 42:780-7. 10.1111/ijlh.13291

15. Zhao Y, Yu C, Ni W, Shen H, Qiu M, Zhao Y: Peripheral blood inflammatory markers in predicting prognosis in patients with COVID-19. Some differences with influenza A. J Clin Lab Anal. 2020, 22:e23657. $10.1002 /$ jcla. 23657 